Gerhard Litscher

\title{
EIN PROJEKTKURZBERICHT ZUR DYNAMISCHEN PULSREAKTION IN COVID-19-PANDEMIE-ZEITEN
}

\author{
IMON/EATCM förderte 2019/2020 als Hauptsponsor RAC-Forschung
}

\section{A Short Project Report on Dynamic Pulse Reaction in COVID-19-Pandemic-Times IMON/EATCM supported RAC-Research as Main Sponsor in 2019/2020}

\begin{abstract}
Zusammenfassung
Der Nogier-Reflex oder Reflex-Auriculo-Cardiac (RAC) bzw. die dynamische Pulsreaktion ist ein physiologisches Phänomen, welches in der Schulmedizin (noch) nicht allgemein akzeptiert wird. Daher ist intensive evidenzbasierte Forschung auf diesem für die Aurikulomedizin so wichtigen Teilbereich notwendig, um entsprechende wissenschaftliche Beweise für eine bessere Akzeptanz bereitzustellen. In diesem kurzen Projektreport wird eine RAC-Messung basierend auf einer modifizierten Smartphone-Registrierung und -Analyse vorgestellt. Der Beitrag soll ForscherInnen stimulieren, weiter auf diesem interessanten Gebiet zu arbeiten.
\end{abstract}

\section{Schlüsselwörter}

Nogier-Reflex, Reflex-Auriculo-Cardiac (RAC), dynamische Pulsreaktion, Aurikulomedizin, Grundlagenforschung, evidenzbasierte Komplementärmedizin

\section{Summary}

The Nogier-Reflex or Reflex-Auriculo-Cardiac (RAC) or the dynamic pulse reaction is a physiological phenomenon which is not (yet) generally accepted in conventional medicine. Therefore, intensive evidencebased research in this sub-area, which is so important for auricular medicine, is necessary in order to provide appropriate scientific evidence for better acceptance. In this short project report, a RAC measurement based on a modified smartphone registration and analysis is presented. The contribution is intended to stimulate researchers to continue working in this interesting area.

\section{Keywords}

Nogier-reflex, reflex-auriculo-cardiac (RAC), dynamic pulse reaction, auricular medicine, basic research, evidence-based complementary medicine

Zusammenarbeit zwischen der Medizinischen Universität Graz, der Initiative für Medizin ohne Nebenwirkungen (IMON e. gemeinnütziger Verein) und der Europäischen Akademie für Traditionelle Chinesische Medizin (EATCM) 2019-2020

D ie Bedeutung des Nogier-Reflexes oder ReflexAuriculo-Cardiac (RAC) bzw. der ,dynamischen Pulsreaktion“ als bisher in der Schulmedizin nahezu unbekannte physiologische Größe kann als erheblich angesehen werden [1]. Es handelt sich um eine dynamische Veränderung [1]. Normalerweise spüren Sie den RAC, während gleichzeitig Mikroreize auf den Patienten einwirken. Dies ist kein statischer Prozess. Sie spüren es nor- malerweise nur mit einer Fingerspitze. Es gibt nur einen idealen Pulsabtastpunkt (in einer anatomisch vorherbestimmten Position). Die RAC-Palpation liefert reproduzierbare Ergebnisse in Doppelblindtests [1].

In einem (2019/2020) multinationalen Forschungsprojekt des TCM (Traditionelle Chinesische Medizin) Forschungszentrums an der Medizinischen Universität Graz, gefördert durch die IMON/EATCM, war man nun be- 


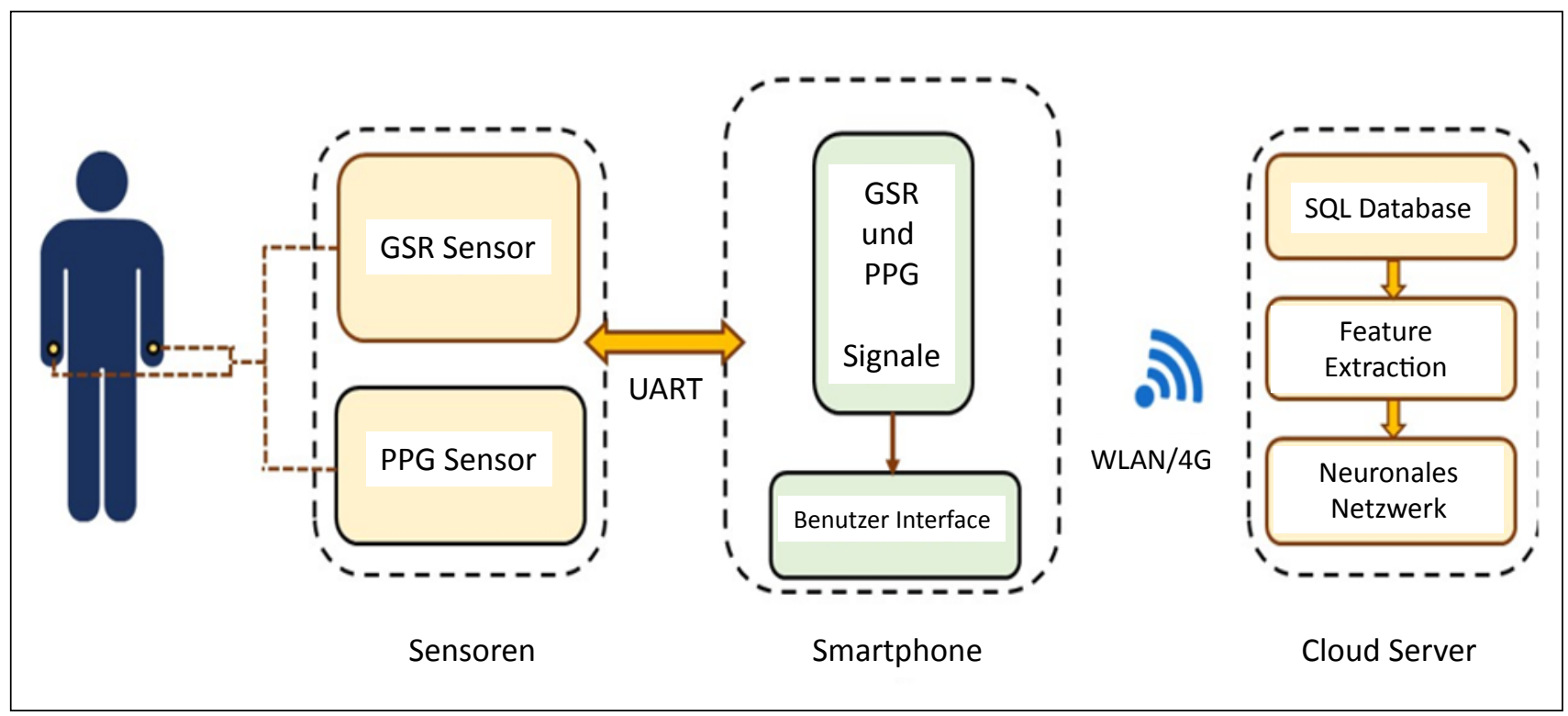

Abb. 1: Systemarchitektur (modifiziert nach [2])

strebt, neue Wege zu gehen, die in erster Linie quantifizierbare Nachweise für die Veränderungen der dynamischen Pulserfassung mit optischen Methoden sowie künstlicher Intelligenz liefern sollen. Die Resultate, welche erstmals mittels eines adaptierten Smartphones registriert wurden, sind als wichtiger Impulsgeber für mögliche weitere Forschungen anderer Forschungsgruppen gedacht.

Im Rahmen dieses Kurzbeitrags soll ein Beispiel eines über ein modifiziertes Mobiltelefon registrierten RAC gezeigt werden, ausführliche technische Beschreibungen finden sich in einem Report, der bereits im Heft 3/2020 dieser Zeitschrift einer breiten Interessengemeinschaft vorgestellt wurde [2].

\section{Informationstechnologisch gestützte dynami- sche „Pulstastung“ mittels adaptierten Smart- phones}

Das von Bahr bezeichnete „dynamische Pulsphänomen“ [1] wurde erstmals in den 1970er-Jahren von Paul Nogier beschrieben [3-5]. Basierend auf der Annahme, dass die meisten Zellen des Organismus auf Reize des autonomen Nervensystems reagieren, suchte Nogier nach einer Methode, mit der die Reaktion des Körpers auf akupunkturinduzierte Maßnahmen dargestellt werden kann. Er entdeckte, dass durch mechanischen Druck auf bestimmte Punkte der Ohrmuschel eine kardiovaskuläre Reaktion stattfand. Er nannte diese Reaktion ,réflexe auriculo cardiaque", später wurde diese auch als RAC abgekürzt oder wie von Bahr bevorzugt mit „dynamische Pulsreaktion“ (zur Abgrenzung von der chinesischen statischen Pulstas- tung) [1] bezeichnet. Bei der Forschung von Bahr u. Mitarb. diente der RAC für seine praktische Anwendung auf dem Gebiet der Aurikulomedizin sowohl als Instrument für Diagnose als auch Therapie [6]. Die Verwendung des RAC-Signals ermöglichte die Entwicklung der Interferenzfeldakupunktur sowie der therapeutischen sogenannten „kontrollierten Akupunktur“, bei der die Akupunkturnadel unter dynamischer Pulssteuerung positioniert wird und nicht wie in der klassischen chinesischen Tradition aufgrund von Cun und anderer Maßeinheiten [6]. Litscher u. Mitarb. [7] veröffentlichten 2014 zusammen mit deutschen und chinesischen Forschern einen Originalartikel in Integrative Medicine International. Eine neue hochauflösende Bildgebungstechnik zur Registrierung pulsierender Oberflächenänderungen könnte es ermöglichen, den RAC zum ersten Mal reproduzierbar auch ohne statistische Analyse zu quantifizieren. Diese Methode kombiniert ein innovatives Mikroskopsystem, eine Videoanalysesoftware und eine spezielle Bildverarbeitungssoftware (von der Pekinger Universität für Wissenschaft und Technologie). Selbst kleine, pulsabhängige Veränderungen der Hautoberfläche konnten deutlich sichtbar gemacht werden [7].Von Moser u. Mitarb. [8], ebenfalls von der Medizinischen Universität Graz in Österreich, wurde 2017 der Beweis veröffentlicht, dass der RAC den physiologischen Gesetzen recht gut folgt. Die Studie baute auf einer früheren Reihe von Tests auf, die auf Messungen und Analysen der Herzfrequenzvariabilität basierten, und zeigte verschiedene reproduzierbare Änderungen physiologischer Parameter. Insgesamt wurden 7 Parameter sowie verschie- 


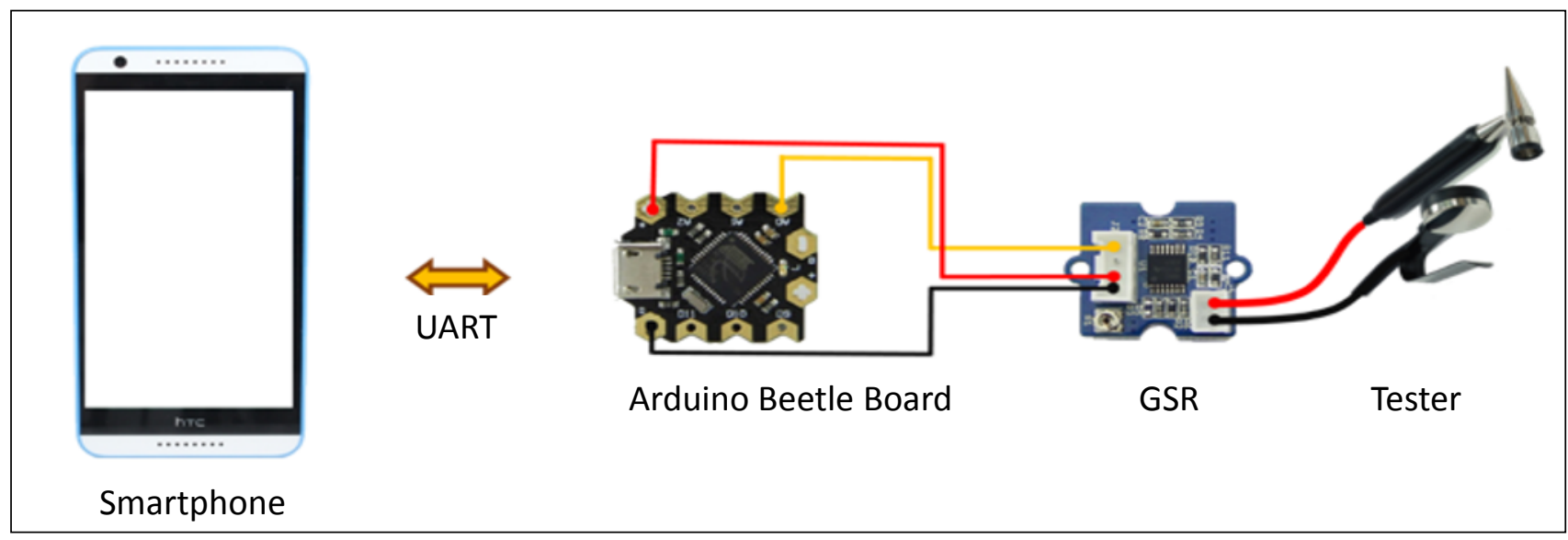

Abb. 2: Der Schaltplan des GSR-Sensors (Galvanic Skin Response; Hautwiderstandsantwort) (modifiziert nach [2])

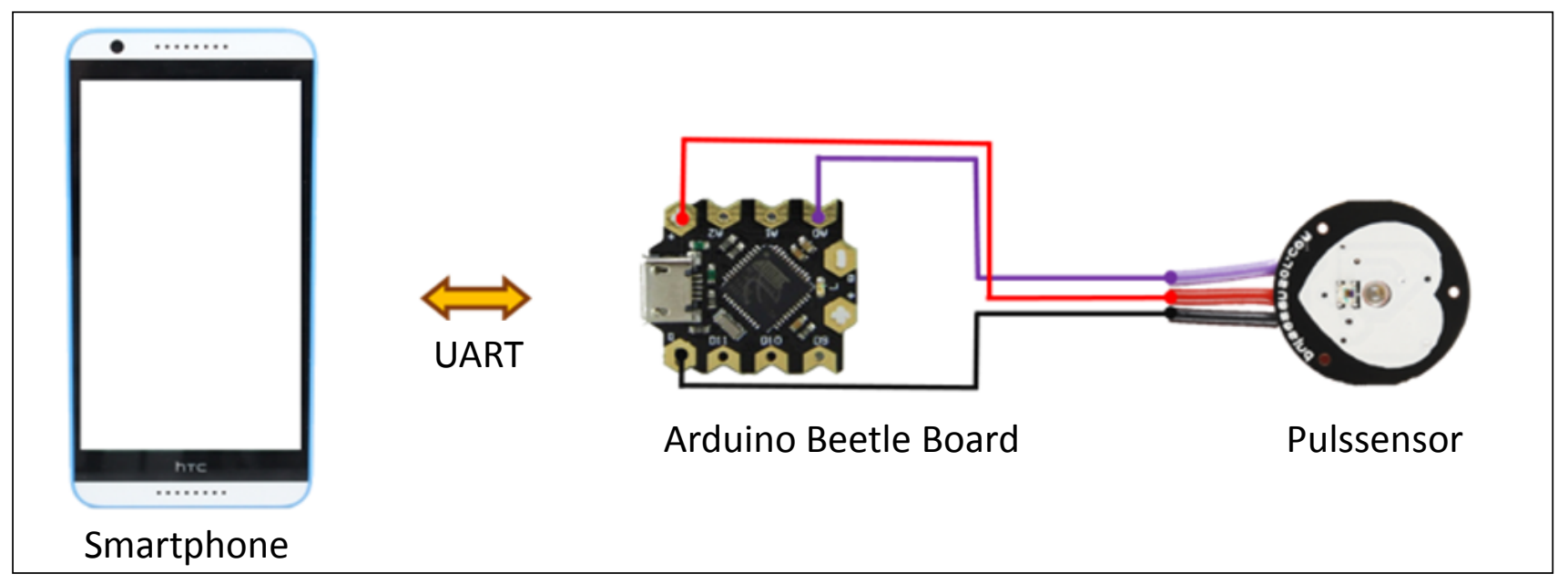

Abb. 3: Der PPG-Pulssensor (PhotoPlethysmoGraphie) (modifiziert nach [2])

dene externe, nicht invasive Stimuli unter einem definierten Setup- und Testkurs aufgezeichnet. Die Autoren nahmen an, dass der RAC eine Manifestation einer Herzreaktion des autonomen Nervensystems ist, die dann eine Pulswelle auslöst. Sie kamen zu dem Schluss, dass die Verwendung subtiler Reize nahe der Wahrnehmungsgrenze angemessen sein sollte, um wiederholbare Tests der unmittelbaren vagalen sowie der verzögerten sympathischen Reaktion zu ermöglichen.

Die in diesem aktuellen Report vorgestellte Technik und Anwendung beruht nun erstmals auf einer modifizierten Smartphone-basierten Registrierung der Pulsaktivität und kann sowohl auf statische als auch auf eben diese dynamischen Pulsreaktionen angewendet werden.

Die Hardwarearchitektur des innovativen Systems ist in der Abb. 1 dargestellt. Sie ist in 3 Teile unterteilt, in die Sensoren, in das Smartphone per se und in den Abschnitt Cloud-Server. Die Sensoren registrieren zunächst GSR(Galvanic Skin Response; Hautwiderstandsantwort) und
PPG-Signale (PPG: Photoplethysmographie) vom Patienten und transferieren sie über das OTG-Kabel (On-TheGo) an das Smartphone. Anschließend überträgt die mobile Einheit die Daten drahtlos an den Cloud-Server. Die Vorhersage des RAC erfolgt mithilfe künstlicher neuronaler Netzwerke.

Abb. 2 zeigt den Schaltplan der GSR-Sensoreinheit, die aus einem Arduino Board, einem GSR-Modul und der Sonde (Tester) besteht. Ein Mobiltelefon wird verwendet, um die GSR-Daten in die Cloud zu übertragen und das Arduino Board über OTG mit $5 \mathrm{~V}$ zu versorgen. Das GSRModul besteht aus einer Brückenschaltung und einem Verstärker. Die Brückenschaltung dient zur Messung des Widerstands und funktioniert ähnlich wie ein Potentiometer. Nachdem der gemessene Widerstand über die Brückenschaltung in eine Spannung umgewandelt wurde, verstärkt der Verstärker die Signale auf die gewünschte Größe. Die verstärkten Signale werden vom ADC (Analog Digital Converter) am Arduino Board gelesen und dann 


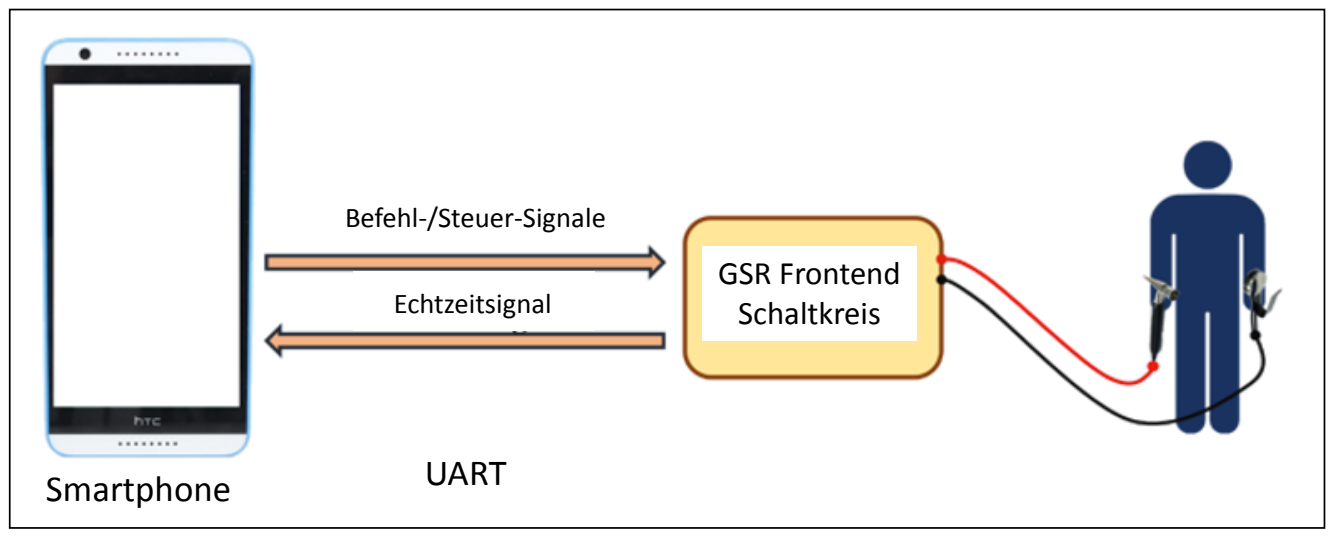

Abb. 4: Kommunikation zwischen GSR-Sensor und Smartphone (modifiziert nach [2])

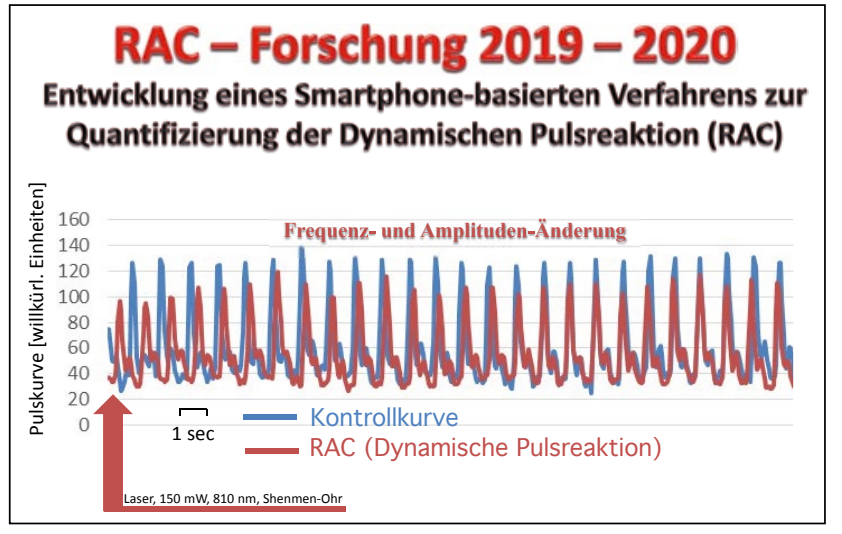

Abb. 5: Dynamische Pulsreaktion (RAC), ausgelöst durch eine Laserstimulation am Ohrpunkt Shenmen

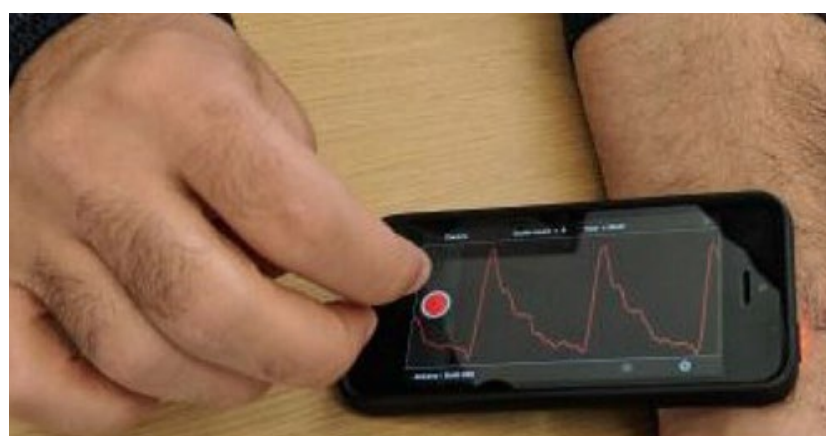

Abb. 6: RAC-Analyse mittels eines adaptierten Smartphone.

über UART (Universal Asynchronous Receiver Transmitter) an das Smartphone weitergeleitet.

Abb. 3 demonstriert den Schaltplan des PPG-Sensormoduls. Ähnlich wie beim GSR-Sensor ist der PPGPulssensor mit einer Arduino Platine verbunden, und das Smartphone wird verwendet, um die PPG-Daten von der Platine zu lesen.

Das vorgeschlagene System ist auf Android Basis implementiert. Wie in Abb. 4 dargestellt, werden sowohl die Steuerpakete als auch die Datenpakete über UART zwischen dem Telefon und dem GSR-Sensor gesendet.
In Abb. 5 ist die dynamische Pulsreaktion (RAC) nach Auslösung durch eine Laserstimulation am Ohrpunkt Shenmen $(810 \mathrm{~nm}, 150 \mathrm{~mW})$ dargestellt. Man erkennt sowohl die Änderungen der Amplitude als auch der Frequenz im Pulssignal der A. radialis deutlich.

\section{Zukunftsaspekte}

Die RAC-Forschung tritt in eine neue Phase ein, in der moderne Informationstechnologie und künstliche Intelligenz (KI) eine tragende Rolle spielen werden. Über adaptierte Smartphones mit integrierten Sensoren und Analysemethoden der KI (Neuronale Netze) könnte der RAC in Zukunft quantifiziert und dokumentiert werden (Abb. 6).

KI wird durch Registrieren, Speichern und Analysieren von RAC-Daten helfen, bessere medizinische Entscheidungen zu treffen. Soweit die Theorie. Doch in der Praxis gestaltet es sich oft schwierig, die Veränderungen der Pulsdaten in anwendbare Informationen umzuwandeln. $\mathrm{Zu}$ unterschiedlich sind die Anforderungen der MedizinerInnnen, zu vielfältig die Einflussfaktoren.

So geht man heute bereits wieder den umgekehrten Schritt und der Faktor Mensch spielt wieder eine größere Rolle. Die aus Sicht des Autors größte Schwierigkeit liegt darin, die menschliche Wahrnehmung in den RAC-Analyseprozess einzubeziehen. Die Lösung dieser Fragestellungen kann nur sein, dass KI und informationstechnologische Prozesse bei der RAC-Forschung nicht Ersatz des Faktors Mensch sein können und sollen, sondern die Basis für dessen datenunterstützte Entscheidungsfindung.

\section{Danksagung}

Der Autor dieses Berichtes möchte sich bei allen Mitgliedern der Initiative für Medizin ohne Nebenwirkungen (IMON e. gemeinnützigerVerein), der EATCM und selbstverständlich ganz besonders bei deren Präsidenten Herrn Professor Frank Bahr herzlich für die an die Medizinische 
Universität Graz geleistete Förderung bedanken. Ein herzliches Dankeschön auch für die Durchsicht des Manuskriptes an Prof. Bahr und an die Projektpartner und MitarbeiterInnen aus Österreich, China und Regionen Chinas, speziell Te-Hsuan Hung, PhD, Kun-Chan Lan, Prof. PhD, beide Department of Computer Science and Information Engineering, National Cheng Kung University, Tainan, Taiwan und Lu Wang, Priv.-Doz. MD LAc,

\section{Literatur}

[1] Bahr F. Persönliche Mitteilungen 8.7. und 22.10.2020.

[2] Litscher G (ed.). Dynamic pulse reaction and auricular medicine. Preliminary results from innovative transcontinental developments and basic research. Project report including 10 peer reviewed manuscripts. Graz:TCM Research Center Graz; 2020 .

[3] Nogier P. Le Réflexe Auriculocardiaque (RAC). Paris: Lyon Méditeranée Medical; 1972; 8: 1709-1738.

[4] Nogier R. How did Paul Nogier establish the map of the ear? Med Acupunct 2014; 26: 76-83.
Research Unit for Complementary and Integrative Laser Medicine, Research Unit of Biomedical Engineering in Anesthesia and Intensive Care Medicine, und TCM Research Center an der Medizinischen Universität Graz, Österreich.

Interessenkonflikte: Der Autor erklärt keinen Interessenkonflikt.
[5] Oleson T.Auriculotherapy stimulation for neuro-rehabilitation. NeuroRehabilitation 2002; 17: 49-62.

[6] Bahr F, Strittmatter B. Das große Buch der Ohrakupunktur. 2. Aufl. Stuttgart: Karl F. Haug Publisher; 2014.

[7] Litscher G, Bahr, F, Litscher D et al. A new method in auricular medicine for the investigation of the Nogier reflex. Integr Med Int 2014; 1: 205-210.

[8] Moser M, Frühwirth M, Messerschmidt D et al. Investigation of a micro-test for circulatory autonomic nervous system responses. Front Physiol 2017; 8: 448. doi:10.3389/ fphys.2017.00448.

\section{Erklärung der IMON/EATCM zur vorliegenden Veröffentlichung von Herrn Prof. Dr. Litscher}

„Die RAC-Forschung tritt in eine neue Phase ein, in der moderne Informationstechnologie und künstliche Intelligenz (KI) eine tragende Rolle spielen werden." Dies ist der Schlüsselsatz im vorliegenden Artikel über einen neuen vielversprechenden Ansatz zur Registrierung der Dynamischen Pulstastung (RAC). Wir kennen die hochinteressanten Arbeiten von Prof. Dr. Litscher et al. zum RAC-Nachweis mit einem innovativen Mikroskopsystem und speziellerVideosoftwareanalyse sowie dem Bildbearbeitungsprogramm der Beijing University of Science and Technology [7].
Jetzt kommt ein wichtiger - ja wesentlicher - Schritt, nämlich vom hochkomplexen Laborsystem zur späteren Anwendung mit live-Demonstration in unseren Seminaren. Noch ist es nicht soweit, aber wir dürfen uns schon darauf freuen!

\section{F. Bahr}

mit bestem Dank an unsere ZAA-Redaktion, die es durch zusätzliche Seiten in dieser Heftausgabe ermöglichte, diesen o.e. Beitrag noch aktuell im Jahr 2020 zu publizieren.

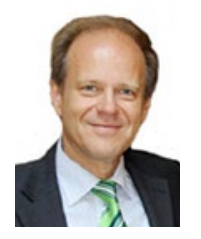

Univ.-Prof. Prof. mult. DI DDr. Gerhard Litscher

Leiter der Forschungseinheit für Biomedizinische Technik in Anästhesie und Intensivmedizin, der Forschungseinheit für Komplementäre und Integrative Lasermedizin

Chairman des TCM Forschungszentrums Graz, Medizinische Universität Graz

Auenbruggerplatz 39, EG19, 8036 Graz, Österreich, E-Mail:gerhard.litscher@medunigraz.at 